\title{
Reef communities show predictable undulations in linear abundance size spectra from copepods to sharks
}

\author{
Freddie Heather ${ }^{1}$, Rick Stuart-Smith ${ }^{1}$, Julia Blanchard ${ }^{1}$, Kate Fraser ${ }^{1}$, and Graham Edgar ${ }^{1}$ \\ ${ }^{1}$ University of Tasmania Institute for Marine and Antarctic Studies
}

March 10, 2021

\begin{abstract}
Amongst the more widely accepted general hypotheses in ecology is that community relationships between abundance and body size follow a log-linear size spectrum, from the smallest consumers to the largest predators (i.e., "bacteria to whales"). Nevertheless, most studies only investigate small subsets of this spectrum, due to extreme size classes that deviate from these linear expectations. In this study, we fit size spectra to field data from 45 rocky and coral reef sites along a 28 latitudinal gradient, and spanning 11 orders of magnitude in body size, from 3 vं $\dot{j}$ to $150 \mathrm{~kg}$. We found that $97 \%$ of the variation in abundance along this 'extended' size gradient was described by a single linear function across all sites. Moreover, consistent 'wobbles' were also observed, with subtle peaks and troughs in abundance along the spectrum, that related strongly to sea temperature and local site conditions.
\end{abstract}

\section{Introduction}

The body size of an organism is often regarded as the single most important factor determining how it interacts with its environment (missing citation). At the community level, the relationship between an individual's body size and abundance can provide important insights into how energy, and hence biomass, moves through the food chain (missing citation); (missing citation). Similar biomass across logarithmic body size classes is often observed in marine communities (missing citation), which equates to decreasing abundance with increasing body size, termed the abundance size spectrum (missing citation).

In marine communities, the faunal abundance size spectrum is often described by a linear function on the log$\log$ scale. The intercept and slope of this function can provide information about nutrient availability (missing citation); (missing citation), human disturbance (missing citation); (missing citation); (missing citation); (missing citation) and feeding strategies of the individuals (missing citation) in the community. In pelagic systems, a consistent size spectrum is commonly observed, often attributed to strict size-based predation (missing citation) and trophic level inefficiencies (missing citation), in combination with the relationship between body size and metabolic rate (missing citation).

Deviations from size spectrum linearity, for example peaks of abundance at specific body sizes, have been described in lake systems (missing citation), intertidal (missing citation) and subtidal (missing citation) benthic communities. (missing citation) attributed these peaks in abundance to the physical environment, whereby sediment grain size would create size-based habitat niches. (missing citation) showed a similar pattern on coral reefs, whereby habitat complexity was shown to drive deviations from linearity via habitat refugia for prey. These abundance peaks have also been attributed to trophic interactions, with early studies proposing the hypothesis of peaks in abundance corresponding to interacting functional groups (missing citation). More recent work has shown mechanistically that these peaks in abundance can arise from bottom-up 
(e.g., food limitation) and top-down (e.g., predation mortality) trophic cascades (missing citation); (missing citation); (missing citation). Whilst a combination of these influences is likely, there is no clear consensus on the drivers of these nonlinear patterns in faunal size spectra.

Reef studies tend to focus on fishes, where observed size spectra are often unimodal, with a peak in abundance at a small to intermediate body size (missing citation). Due to the theoretical expectations of decreasing abundance with body size, and the potential for under-sampling smaller individuals, many reef size spectra studies have routinely excluded individuals less than the modal size, or equivalent mass, from the linear modelling analyses (missing citation); (missing citation); (missing citation); (missing citation)(See Figure 1A). Ignoring the small fishes and fitting a linear model to the size spectrum has the benefit of simplicity and has also been shown to be useful in detecting fishing pressure on reefs (missing citation). These studies have typically used visual survey methods to collect data, which are known to under-represent densities of some species (missing citation), particularly for smaller (missing citation), more cryptic (missing citation); (missing citation) or nocturnal fishes (missing citation). For example, (missing citation) found their visual survey methods underestimated the abundance of reef fishes $<5 \mathrm{~cm}$ by $75 \%$.

In this study we use individual body size data spanning $3 \mu \mathrm{g}$ to $150 \mathrm{~kg}$ across $28^{\circ}$ of latitude to systematically test the a) generality of a linear size spectrum on reefs, and b) the presence and cause of the dip in abundance at the small to medium size classes. We used two distinct methods to collect field data on abundance and size of consumer taxa on 45 reefs from $14.7^{\circ} \mathrm{S}$ to $43.3^{\circ} \mathrm{S}$ on the eastern coastline of Australia, including macroalgal covered temperate rocky reefs and coral reefs in the tropics. One method involved collection sampling of animals associated with benthic habitat ("epifauna": 0.125 - $22 \mathrm{~mm}$ body size), while the other involved visual censusing of larger mobile invertebrates and fishes ("visual survey data" 0.01 - $4 \mathrm{~m}$ body size) along underwater transect lines. Together these approaches provided density estimates for all species that could readily be surveyed by divers at a given patch of reef, spanning 11 orders of magnitude in body size.

The inclusion of invertebrates in smaller and overlapping size classes to the fishes allows for testing of three hypotheses about causes of the abundance dip in the size spectrum observed in reef fishes (Figure 1A): 1) It arises from disproportionate under-sampling of small fishes in visual census surveys, as has been assumed in previous studies and formed the basis for removal of this part of the spectrum. This would be seen through a 'gap' in the size spectrum (Figure 1B); 2) It is part of a consistent curve in the overall size spectrum of reef consumers, which would be seen in a continuous, but curved size spectrum (Figure 1C); 3) It is an artefact of only considering fishes in isolation, and that part of the spectrum is filled by invertebrates that are usually neglected (missing citation). This would be seen by a strongly linear overall spectrum (Figure 1D). These competing hypotheses each assume the overarching principles of size-based feeding (missing citation) and transfer inefficiency (missing citation) are operating in reef systems, which have been well supported by previous studies (missing citation).

\section{Methods}

\section{Survey data}

Data collection was performed using two distinct methods: 1) Collection of benthic habitat samples with associated invertebrate epifauna and, 2) underwater visual surveys of fishes and large mobile macro-invertebrates.

Epifauna were sampled from 45 sites spanning the eastern coast of Australia, from tropical (Lizard Island; $14.7^{\circ} \mathrm{S}$ ) to temperate (Southern Tasmania; 43.3 ${ }^{\circ} \mathrm{S}$ ) reefs, between the years of 2015 and 2018. Sample collection involved firstly characterising the habitat at the site by taking 20 evenly spaced photographs of benthos and substrata along a $50 \mathrm{~m}$ survey transect. Photographs were taken from approximately 50 $\mathrm{cm}$ above the substrata to depict approximately $30 \times 30 \mathrm{~cm}$ of seabed. These photographs were assessed to derive an estimate of the relative abundance of different habitat types at each site. Habitat selected for 
sampling was then covered with a $25 \times 25 \mathrm{~cm}$ grid-marked quadrat and photographed in situ to quantify its planar area. Habitat and epifaunal samples were bagged in situ after detachment by removing soft habitat (e.g., macroalgae, sponges) with a knife and hard coral habitat with a chisel. Habitat that could not easily be removed (e.g., turfing algae, encrusting coral) was vacuum sampled using a venturi air-lift. Each habitat sample was flushed with freshwater to remove mobile epifauna, which were then passed through a set of logarithmic $(\log$ base $=\sqrt{2})$ mesh size sorting sieves. Animals retained on each sieve were counted and identified to the highest possible taxonomic resolution. For more detailed methodology see (missing citation). Abundance of epifauna by size and taxa were standardised to $1 \mathrm{~m}^{2}$ planar area, by accounting for the area of each sampled habitat unit.

Fish and large mobile invertebrate species ( $>2.5 \mathrm{~cm}$ maximum recorded length) were surveyed using the standardized Reef Life Survey visual census methods (missing citation); (missing citation), in which SCUBA divers swim along a $50 \mathrm{~m}$ transect line and record all fishes and invertebrates observed within $5 \mathrm{~m}$ and $1 \mathrm{~m}$ wide belts, respectively. Divers estimate body size of animals observed to the closest of 13 size categories $(2.5,5,7.5,10,12.5,15,20,25,30,35,40,50$, and $62.5 \mathrm{~cm})$ or to the nearest $12.5 \mathrm{~cm}$ for body sizes greater than $62.5 \mathrm{~cm}$. For large mobile invertebrates, of which body size was not always estimated at the time of observation, body size was estimated using the lognormal probability distribution of body size based on the asymptotic size of the species (missing citation). Densities of fishes and invertebrates were standardised to abundance per $\mathrm{m}^{2}$ by dividing the individual number counts by the respective area surveyed.

\section{Weight estimation}

Epifaunal ash-free dry weight (AFDW) was estimated using published length-weight conversions (missing citation). We note that these published conversions do not account for animals $<0.5 \mathrm{~mm}$, however following linear extrapolation of well supported trends (missing citation) we assume the conversions also apply to smaller individuals. Wet weight (WW) was estimated as WW $=$ AFDW/0.22 (missing citation).

From the visual survey data, an individual's body mass was estimated from body length using published species-level length-weight relationships (missing citation); (missing citation) following the methods of (missing citation), or, where species-level information was not available, an overall length-weight relationship for the taxonomic class.

\section{Combining the datasets}

The body masses of individuals from the two datasets overlapped. To combine these data, we binned both datasets into log bins with a base of 2.5 and summed the abundance of the bin. A log base of 2.5 was chosen as it represented the smallest reasonable log bin base that would not result in double binning (i.e., two bins combining into a single bin) in either of the two datasets. Normalised density was calculated as the density (abundance per $\mathrm{m}^{2}$ ) divided by the width of the body size bin (missing citation).

\section{Fitting nonlinear size spectra}

Due to clear sinusoidal patterns in the residuals of linear functions between log body mass and log abundance, we fitted a nonlinear size spectrum model proposed by (missing citation) (Equation 1). This model included both a linear function with an additional sinusoidal function to allow for the quantification of both the linear and secondary structure aspects of the size spectrum.

$$
\log (N)=\beta_{0}+\lambda \cdot \log (M)+A \cdot \sin \left(\frac{2 \cdot \pi \cdot \log (M)}{D}-P\right)
$$


Where, $\mathrm{N}$ is the normalised density $\left(\mathrm{m}^{-2}\right), \mathrm{M}$ is the middle of the mass bin $(\mathrm{g}), \beta_{0}$ is the size spectrum intercept, $\lambda$ is the slope, and $\mathrm{A}, \mathrm{D}$ and $\mathrm{P}$ represent the amplitude, wavelength, and phase of the sine wave, respectively. The size spectrum model (Equation 1) was fitted using the 'nlrq' function (missing citation) in $\mathrm{R}$ (missing citation). The amplitude (A) represents the 'strength' of the sine wave, and therefore the deviation from linearity in the size spectrum. (missing citation) used the pareto distribution to detect deviations from linearity, due to the body size data being resolved to the individual level rather than in logarithmic size bins. Individual level body size data would allow for the detection of finer-scale deviations from linearity, however, this was not feasible in this study due to the inherent binning nature of data collection (both sieving and visual survey methods). The ratio of abundance from the top of one peak to the bottom of a trough can be calculated as the log base to the power of two times the amplitude $\left(2.5^{2 A}\right)$. The body mass ratio of individuals occupying neighbouring peaks can be calculated as the log base to the power of the wavelength $\left(2.5^{D}\right)$, and the distance between consecutive peaks and troughs can therefore be estimated as the log base to the power of half the wavelength $\left(2.5^{\frac{D}{2}}\right)$.

Due to the difficulty of interpreting the phase parameter $(\mathrm{P})$ of the nonlinear size spectrum when the wavelength (D) is not fixed (missing citation), we identified the body mass in which local peaks and troughs occurred in the size spectrum model using the 'optimize' function in R (missing citation).

\section{Environmental covariates}

We fitted a series of maximal linear models (Supplementary material S4) to identify the most important covariates in estimating the parameters of the size spectrum model (Equation 1). These site covariates included mean sea surface temperature $\left({ }^{\circ} \mathrm{C}\right)$, mean chlorophyll level $\left(\mathrm{mg} \mathrm{m}^{-3}\right)$, phosphate and nitrate levels $\left(\mathrm{mmol} \mathrm{l}^{-1}\right)$, all extracted from Bio-ORACLE, (missing citation), and categorical indices of wave exposure, habitat relief, currents, and reef floor slope, scored on a 1 to 4 scale by divers at the survey sites. Details on these environmental variables can be found in Supplementary S2. Using only the covariates with a significant effect $(P<0.05)$, we then fit a simplified linear regression model to each model parameter (Table S4.1).

\section{Results}

Despite the two distinct methods of data collection and the extrapolation of epifauna data from the scale of individual habitat samples through to the transect scale, combining the two datasets resulted in a continuous size spectrum in all sites (Figure 2). Whereby log body mass (M) described $97 \%$ of the variation in $\log$ normalised abundance $(\mathrm{N})$ (linear regression: $\log (\mathrm{N}) \sim \log (\mathrm{M})$; all sites combined). More variation was explained when combining these datasets (Adjusted $\mathrm{R}^{2}=96.7 \%$ ), than when a linear model was fitted to the datasets separately (Epifaunal data, Adjusted $\mathrm{R}^{2}=95.1 \%$; Visual survey data, Adjusted $\mathrm{R}^{2}=78.8 \%$ ) (Figure 1).

Clear sinusoidal patterns were present in the residuals of the linear model fits (Equation S1.1) (Figure 3). At all 45 sites, the nonlinear size spectrum model (Equation 1, Figure S1.1) provided a better fit than a linear model (Equation S1.1, Figure S1.1), as determined by the Akaike information criterion (missing citation). The mean community level predator-to-prey mass ratio (PPMR), calculated as the log base to the power of half the wavelength $\left(2.5^{\frac{D}{2}}\right)$, was estimated to be $5800\left(10^{3.76}\right)$, with a range of $430\left(10^{2.63}\right)$ to $93000\left(10^{4.97}\right)$.

Wave exposure and mean sea surface temperature (SST) explained $34 \%$ of the variance in the size spectrum slope. Sites with greater SST and wave exposure had shallower size spectra. Wave exposure and SST also explained $40 \%$ of the variation in the position of the local peak abundance, whereby the peak abundance occurred at around $630 \mathrm{~g}$ in temperate sites compared to around $60 \mathrm{~g}$ at tropical sites. 
The slope of the reef floor explained $25 \%$ of the variation in the amplitude, where a steeper reef floor created more linear size spectra (smaller amplitude), and $13 \%$ of variation in the wavelength, where sites with steeper reef floor had a shorter wavelength (i.e. a smaller calculated PPMR).

\section{Discussion}

Whilst there was an extremely strong linear component to the size spectra, the addition of a sinusoidal component resulted in a better model fitting at all 45 sites, and clearly supported our second hypothesis (Figure 1C); that the abundance dip commonly assumed to be solely a sampling artefact, is part of a continuous, nonlinear size spectrum. A few sites nevertheless provided clear evidence of under-sampling (e.g., KG-S13, KG-S15 and SYD31 in Figure 2). Thus, some support for the first hypothesis (Figure 1B) also exists, with under-sampling of smaller sized fishes associated with the fact that the visual methods cannot cover fishes with equal probability of observation along the size spectrum. Yet the data suggest that under-sampling is not likely the primary reason for the non-linearity in reef fish size structure, potentially affecting conclusions of previous studies where smaller size classes had been removed.

The slope $(\lambda)$ estimates from the nonlinear model here ranged from -2.00 to -1.52 with a mean slope of -1.72 , roughly consistent with previous reef studies (equivalent normalised abundance size spectrum slope values: (missing citation); (missing citation); (missing citation), (missing citation)), and near identical to the estimate of -1.75 that would be expected by the energy equivalence rule for situations with individuals feeding on a common resource (i.e. herbivory-dominated) (missing citation). Notably, however, these previous studies fit a linear model to the descending limb only (missing citation). The removal of the individuals in the abundance dip likely has the effect of steepening the size spectrum slope; for example, data from (missing citation) estimate a mean slope of -2.05 for the same 45 sites when considering the descending limb only (individuals $>32 \mathrm{~g}$ ). Thus, we must recognise that the slopes of linear size spectra fitted only to the descending limb are potentially steeper that those of the broader scale size spectrum.

The dip in abundance in the range of $0.001 \mathrm{~g}$ to $10 \mathrm{~g}$ for visual census data observed here (blue datapoints in Figure 2, see also Figure 1A), is consistent with the patterns observed in previous reef size spectra studies (missing citation); (missing citation). Two lines of evidence suggest this dip is unlikely a sampling bias: 1) (missing citation) used rotenone poisoning sampling to comprehensively sample all reef fauna and observed a similar dip in abundance in this size range. 2) The dip in abundance appears to be a true feature in the overall size spectrum (Figure 1) that is continuous and nonlinear (i.e., supporting $\mathrm{H}_{2}$, Figure 1C).

Wave-like patterns in size spectra have been previously observed in lake studies (missing citation); (missing citation), and have been reproduced in mechanistic modelling studies (missing citation); (missing citation). These mechanistic approaches have shown trophic cascades driven by fishing pressure (missing citation) and nutrient enrichment (missing citation) can result in wave-like patterns in size spectra. Both studies found that a combination of bottom-up (food availability) and top-down (predation mortality) pressures drive the observed patterns. Further, both studies found wave amplitude to increase with body size, i.e., greater linearity in the size spectrum at smaller body sizes; similar to the patterns observed here (Figure 2). This could be formally tested with a model that allows amplitude to vary with body size, however this was not applied here due to potential for overparameterization of the model (missing citation). A test to assess whether nutrient enrichment drives sinusoidal patterns in lakes (missing citation) requires a greater range of available nutrients (phosphates and nitrates) than was available at the reef sites in this study. If the sinusoidal patterns observed in this study are driven by trophic interactions, we would expect the distance between peaks and troughs to correspond to a mean predator-to-prey mass ratio (PPMR) at the community level. Using the wavelength to calculate PPMR, we estimated a mean community PPMR of $10^{3.76}$, which is consistent with previous literature (Trebilco et al 2013), further supporting the hypothesis that these sinusoidal patterns are driven by trophic interactions.

Another theory explaining nonlinearity in size spectra assumes habitat complexity provides refugia that 
favour particular body sizes (missing citation). We observed no significant relationship between the survey site relief (a broad-scale measure of habitat complexity) and the size spectrum amplitude (A in Equation 1; a measure of nonlinearity). There are several possible reasons for this. Firstly, site relief was classified categorically into four levels to describe the broad-scale habitat structure, but these categories did not encompass the scale of crevices likely to be used as prey refugia. Secondly, wave amplitude is a measure of the strength of the sine curve, not necessarily a measure of fine-scale deviations from linearity that would be expected from fine-scale habitat complexity. Complex habitats are likely to provide a wide range of refugia of varying scales (missing citation); (missing citation); (missing citation); (missing citation) and associated potential niches, and therefore likely to produce multiple peaks and troughs in the size spectrum rather than a consistent sinusoidal wave. Further work into the residuals of the current nonlinear model could identify a "tertiary structure" to the size spectrum, potentially caused by finer-scale drivers, such as habitat niches and refugia.

Environmental variables explained a large portion of variability in the size spectra; both the linear $(\lambda)$ and nonlinear components (A, D, and position of peak abundance). Figure 3 shows that the peak in abundance appears at larger body sizes in cooler sites compared to warmer sites: we estimate the peak in body sizes to increase by an order of magnitude from $26^{\circ} \mathrm{C}$ to $14^{\circ} \mathrm{C}$ waters $(60 \mathrm{~g}$ to $630 \mathrm{~g}$ body mass). These peaks approximately correspond to the mean body mass of the dominant large invertebrate-feeding fishes in temperate regions (e.g. wrasses) and the sometimes hyper-abundant small planktivorous fishes on coral reefs. Numerous explanations potentially account for latitudinally-dependent body size preference. Firstly, the dominant energy pathways may vary with latitude, whereby a higher mean PPMR in temperate reefs leads to less energy lost through trophic inefficiencies and therefore a peak in abundance at larger body sizes. Habitat composition, which varies latitudinally, will also likely play an important role in preferential body sizes (missing citation); (missing citation). Complex habitat structure, for example crevices of varying sizes in coral reefs, provides a diversity of niches and therefore drive competition for space and resources, potentially leading to a smaller average body size.

Size spectra are widely used as ecological indicators of reef health, for detecting and quantifying ecosystem disturbances such as fishing pressure (missing citation); (missing citation); (missing citation); (missing citation). This application of empirical size spectra as ecological indicators often relies on the assumption that relationships between log abundance (or biomass) and log body size are linear (missing citation); (missing citation); (missing citation). Here, we show that $97 \%$ of the variation in log abundance can be explained by a linear function of log body mass in individuals ranging from $3 \mu \mathrm{g}$ to $150 \mathrm{~kg}$, irrespective of taxonomy or location. This detailed empirical support for consistency of marine size spectra supports the generality of early conjectures of linear size spectra holding from "bacteria to whales" (missing citation). However, in order to use size spectra as ecological indicators for reefs we must identify a counterfactual baseline representing an 'unimpacted' reef, from which to compare (missing citation); (missing citation). Although we observed remarkable consistency in the linearity of the size spectra, subtle nonlinearities are evident. These sinusoidal nonlinearities are similar to those previously observed (missing citation) and modelled (missing citation) in lake ecosystems. Whether the inflections reflect disturbances to reefs or are an inherent part of reef size spectra remains speculative. While temperature was found here to be a strong driver of sinusoidal patterns, this factor is likely related to multiple interacting direct and indirect effects on body size, including through changes in habitat composition (missing citation). Trophic cascades potentially also contribute to inflections (missing citation); (missing citation). Mechanistic models trained with empirical data are needed to test this hypothesis and to identify the main drivers of the nonlinear patterns. Data presented here for remote highly protected reefs (e.g. Middleton Reef) provide a baseline for reef size spectra, and for their expanded use as ecological indicators of reef health. 


\section{Acknowledgements}

This research was supported by the Marine Biodiversity Hub, a collaborative partnership supported through funding from the Australian Government's National Environmental Science Program, and used the NCRISenabled Integrated Marine Observing System (IMOS) infrastructure for database support and storage. This research was also made possible by funding of the Australian Research Council (ARC), and the data from the Reef Life Survey Foundation. The authors also thank data support from Antonia Cooper, Just Berkhout, Scott Ling, Ella Clausius and Elizabeth Oh.

\section{Figures}

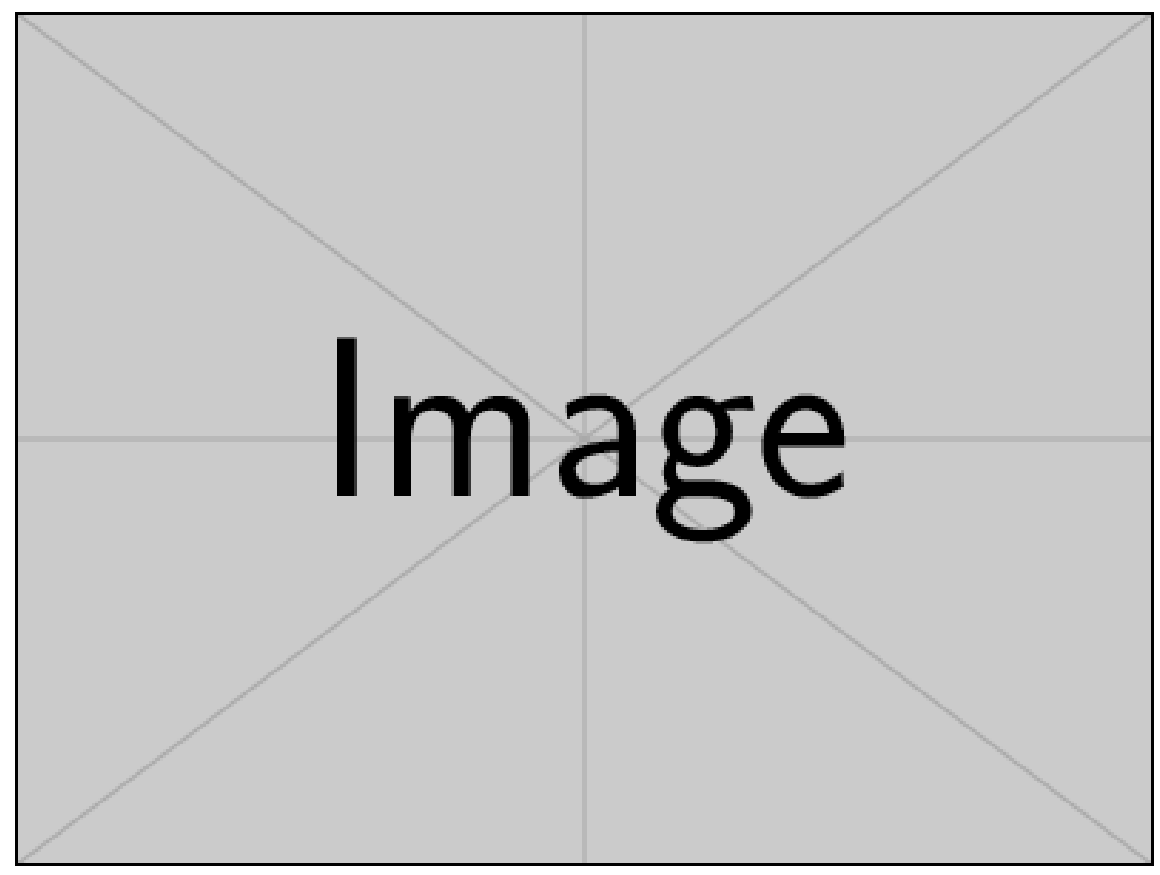

Figure 1: Conceptual diagram showing the dip in abundance of small fishes typically observed in studies of reef fish size structure (A), and three hypothetical spectra (B-D) that would indicate alternative scenarios for the cause of the pattern observed in (A). Where the size spectrum is unimodal (A), a linear function is generally fitted to the descending limb (darker blue), with smaller size classes assumed to be under-sampled (lighter blue) and excluded. A linear overall size spectrum with a substantial gap (B) would indicate undersampling assumption is likely be the cause, while a continuous curve with a smooth transition through the size classes where the fishes and invertebrates overlap (C) would suggest ecological interactions drive a real non-linearity. A continuous strongly linear spectrum (D) would indicate that epifaunal invertebrates perfectly fill the gaps left by fishes. 


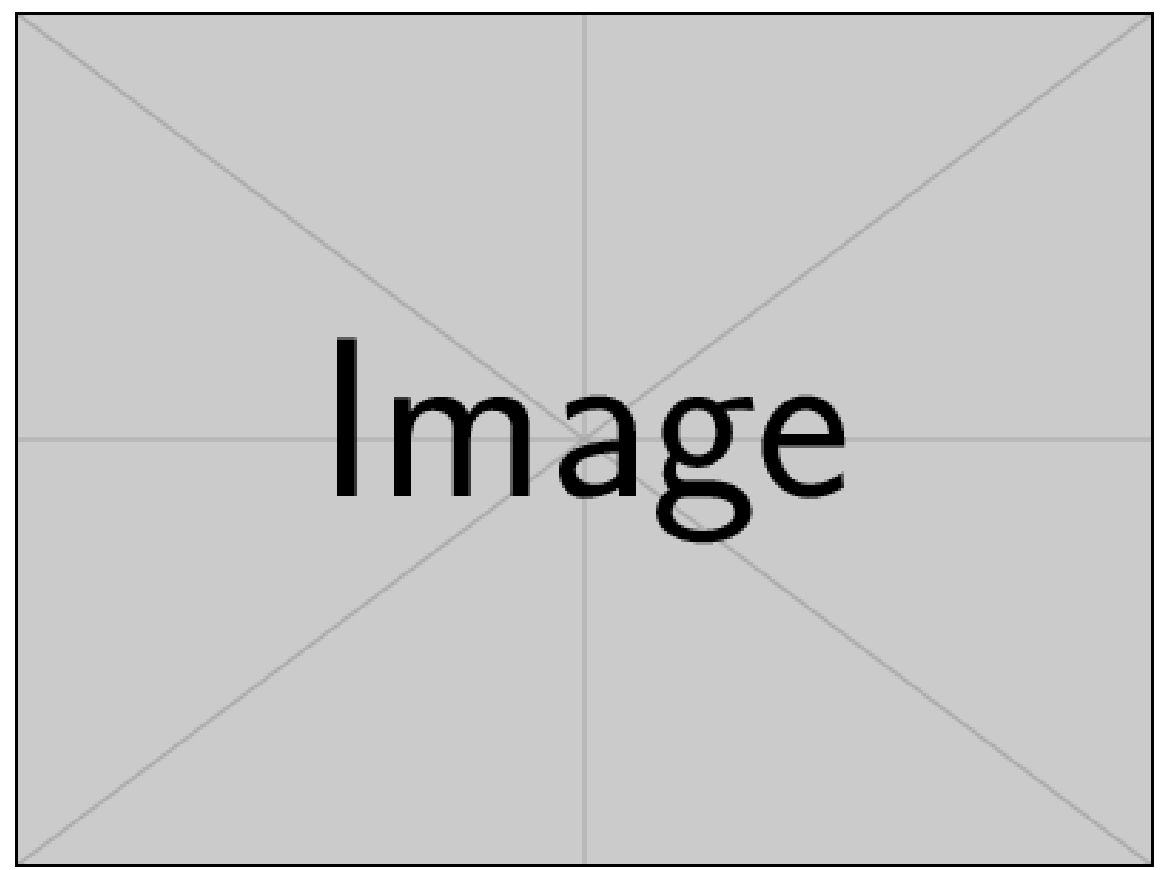

Figure 2: Size spectra for rocky and coral reef ecological communities covering 11 orders of magnitude in consumer size, from $3 \mu \mathrm{g}$ to $150 \mathrm{~kg}$, at 45 sites along the $28^{\circ}$ latitude along the eastern coast of Australia (sites ordered by latitude). Yellow data points represent data from epifaunal sampling, blue data points represent individuals from visual surveys and red data points represent mass bins that contain animals from both sampling methods. 


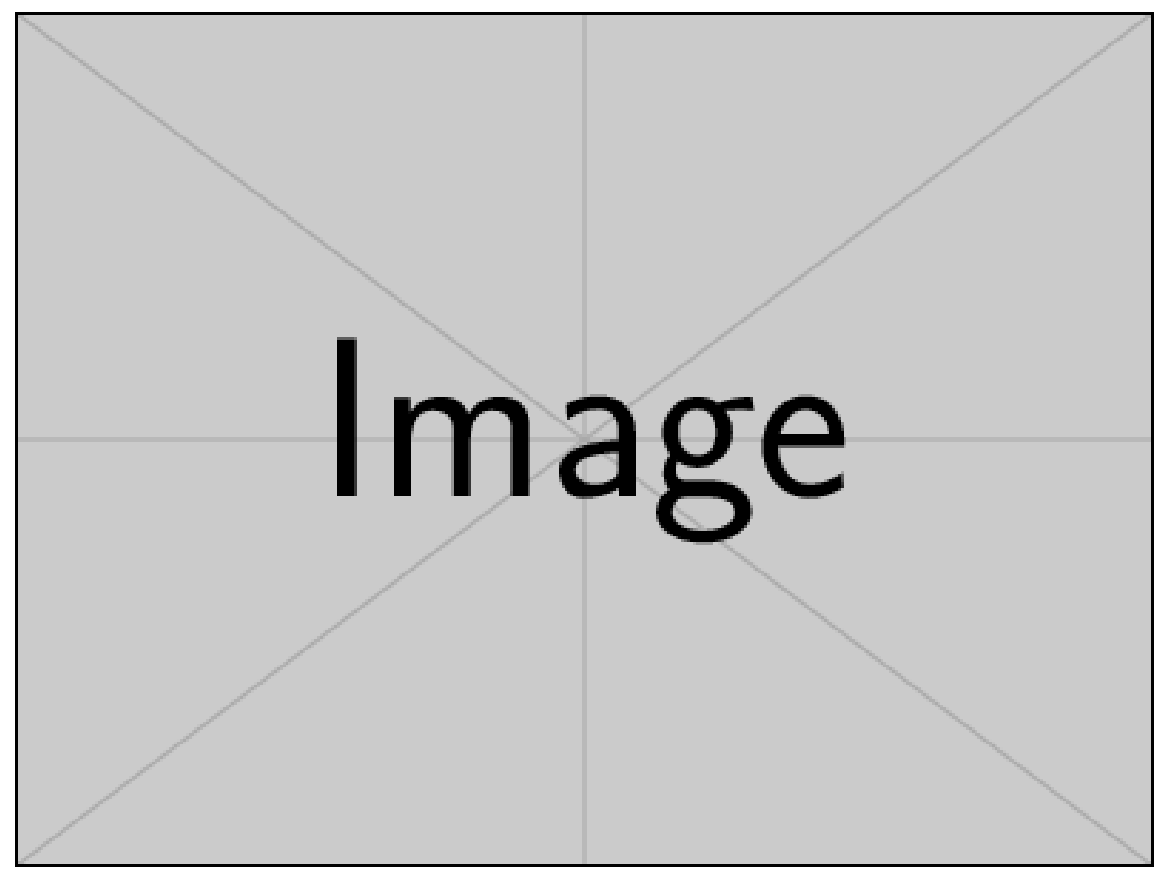

Figure 3: Sinusoidal patterns in the residuals of the linear model of the size spectrum. Each line represents a LOESS fit for a given site. Each thin line represents a site, with the colour of line representing the mean annual sea surface temperature (SST) at the site. The two thick lines represent the combined LOESS fit for tropical (red) and temperate sites (blue).

\section{References}

